This paper is published in the open archive of Mid Sweden University

DIVA http://miun.diva-portal.org

by permission of the publisher

Mårten Sjöström, Sylvain Tourancheau, Xusheng Wang and Roger Olsson, "A locally content-dependent filter for inter-perspective anti-aliasing," Three-Dimensional Image Processing (3DIP) and Applications II, Atilla M. Baskurt; Robert Sitnik, Editors, Proc. SPIE, Vol. 8290, 829006 (2012).

http://dx.doi.org/10.1117/12.908627

(C) Copyright 2012 Society of Photo-Optical Instrumentation Engineers. One print or electronic copy may be made for personal use only. Systematic electronic or print reproduction and distribution, duplication of any material in this paper for a fee or for commercial purposes, or modification of the content of the paper are prohibited. 


\title{
A locally content-dependent filter for inter-perspective anti-aliasing
}

\author{
Mårten Sjöström*, Sylvain Tourancheau, Xusheng Wang, Roger Olsson \\ Dept. of Information Technology and Media, Mid Sweden University, SE-85170 Sundsvall, Sweden
}

\begin{abstract}
Presentations on multiview and lightfield displays have become increasingly popular. The restricted number of views implies an unsmooth transition between views if objects with sharp edges are far from the display plane. The phenomenon is explained by inter-perspective aliasing. This is undesirable in applications where a correct perception of the scene is required, such as in science and medicine. Anti-aliasing filters have been proposed in the literature, and are defined according to the minimum and maximum depth present in the scene. We suggest a method that subdivides the ray-space and adjusts the anti-aliasing filter to the scene contents locally. We further propose new filter kernels based on the ray space frequency domain that assures no aliasing, yet keeping maximum information unaltered. The proposed method outperforms filters of earlier works. Different filter kernels are compared. Details of the output are sharper using a proposed filter kernel, which also preserves the most information.
\end{abstract}

Keywords: 3D multiview, inter-perspective aliasing, ray space filtering

\section{INTRODUCTION}

Three-dimensional presentations on multiview and lightfield displays projecting a number of views into a viewing space have become increasingly popular. Although these displays are constantly improving as the technology develops, the restricted number of views implies an unsmooth transition between views if details are far from the display plane. This is undesirable in applications where a correct perception of the scene is required. Therefore, removing this effect is required while retaining the most possible information.

The applications of multiview and lightfield displays have so far mainly been in entertainment and signage, but they have now also found to be useful in areas of science and medicine ${ }^{1}$. The latter have higher requirements on a correct representation of the displayed scene, as an erroneous presentation can lead to misinterpretations; the depiction should rather contain an uncertainty than an error in order to avoid a false impression. Unsmooth transition between adjacent perspective views may be a cause for a faulty impression, with the possible consequence of incorrect interpretation.

Transitions between views where objects "jump" in position between adjacent views are caused by a high degree of details relative to the change in angle between views. The change between views becomes more prominent for details with larger disparity, i.e. at larger distance from the display plane. The phenomenon is explained by high frequency components of such details being aliased due to a small angular sampling rate (few perspective views), and goes under the name inter-perspective aliasing ${ }^{2}$. Two remedies have earlier been proposed in the literature: depth scaling ${ }^{3,4}$ and antialiasing filtering. The former scales the depth at which details are presented and so distorts depth perception. It is, therefore, not adequate in the mentioned applications, which leaves filtering as an appropriate option.

Sampling of the plenoptic function was studied by Chai et al. ${ }^{5}$ who concluded that most captured light field is affected by aliasing due to a limited number of cameras. They proposed a filter sheared in the frequency domain in order to have the most possible details without allowing aliasing in the reconstruction process. Isaksen et al. ${ }^{6}$ identified that details of different depths will cause aliasing when the sampling rate is too low, and that the image cannot be reconstructed without removing details or causing ghosting.

The sheared filter by Chai et a. was extended with a wide aperture filter by Stewart et al. ${ }^{7}$. They so attempted to reduce the blurriness for certain objects by selecting frequency components for a specific depth, and thereby allowing a small amount of aliasing. Zwicker et al. ${ }^{8}$ chose to center objects around the zero-disparity plane, i.e. the display plane, in order to better use the depth budget of the display, and then applied the wide aperture filter so that details remain sharp in the display plane. They so combined the necessary resampling filter with the wide aperture filter, a display reconstruction filter and the typical shape of data into a combined filter. The method of anti-aliasing filtering has also been applied to 
data with the purpose to improve compression rate of video ${ }^{9}$. Ramachandra et al. ${ }^{10}$ extended the anti-aliasing filtering with a sharpening filter in order to compensate for the 3D display's light leakage (cross-talk) between views.

These earlier works on inter-perspective aliasing filtering have considered the full ray-space spectrum when deciding cut-off frequencies for the anti-aliasing filters. A scene with large differences in depths then implies a cut-off frequency that is unnecessarily small for certain parts of the scene, which leads to a resulting image that is more blurry than depth locally requires. In addition, the previous works have selected different parts of the signal spectrum in order to remove the aliased parts. A closer analysis of the signal spectrum reveals a typical shape of the non-aliased parts of the spectrum, which is a consequence of the construction of the multidimensional ray-space system.

In the present work, we suggest a filtering method that subdivides the ray-space and adjusts the filter to the scene contents locally. In this way, we obtain resulting images that contain more details. Furthermore, we propose new filter kernels based on the ray space spectrum that assures no aliasing, yet keeping more frequencies unaltered. This reproduces more informative results, i.e. less blurry resulting multiview images. The filter amplitude characteristics are based on the depth limits within each subdivision. We propose a method to identify the minimal and maximal depths of the scene in each subdivision in the ray-space spectrum, which are used to define the filter amplitude characteristics.

The rest of the paper is organized as follows: Section 2 defines the problem addressed. Section 3 gives an overview of multiview pixels, ray-space sampling and aliasing. Section 4 describes the proposed filtering method. Section 5 explains how the proposed method was evaluated. Section 6 presents and analyses the results. Section 7 concludes the main points of the work.

\section{PROBLEM DESCRIPTION}

The aim of the study is to improve image quality on multiview and lightfield displays for applications that require a truthful presentation. For this purpose, the image presentation shall give an as accurate recreation of the scene from any viewing angle as possible. The goal of the study is

- to improve multiview image quality by identifying a linear filter that avoids inter-perspective aliasing, yet keeping as much image information as possible, and

- to filter original multiview data previously captured, meaning that no over-sampling of the original scene is possible in order to avoid inter-perspective aliasing.

Aliasing due to high frequency contents may occur in any image. Especially images based on computer graphics may exhibit aliasing effects. This kind of aliasing must be dealt with as for any image. We concentrate here on the consequences due to the constitution of the ray-space and its spectrum. The study is limited to displays with a Horizontal Parallax Only (HPO), i.e. no vertical parallax. Data are supposed to be given, meaning that over-sampling by means of view interpolation from multiview data with depth information is not possible. Depth distortion should be kept to a minimum at the viewing distance for which the multiview display has been designed, which excludes depth compression to overcome inter-perspective aliasing. The original data (input) shall further be assumed to have the placement the user requires. I.e. the placement of objects in depth should be up to the user. This implies that the filtering technique may not move objects to a more favorable depth position for anti-aliasing filtering. Finally, the filtering effect an actual multiview display may have on the experience of viewing the data due to optical cross-talk and alike is not taken into consideration.

\section{RAY-SPACE, SAMPLING AND ALIASING}

\subsection{Ray space}

A real world 3D scene constitutes a continuous lightfield described by the plenoptic function. It depends on the position of the point $(x, y, z)$ and the direction of the incoming light $(\theta, \varphi)$, the light wavelength $(\gamma)$ and time $(t)$ : $I=I(x, y, z, \theta, \varphi, \gamma, t)$. An alternative parameterization of position and direction is by the coordinates of two parallel planes at a distance $D$ apart $^{11,12}$ : the position is given by $(x, y)$ and the direction is given by $(u, v)$, see Fig. 1 .

In the present work, we consider images displayed on multiview or light field displays that have a Horizontal Parallax Only (HPO), so the vertical angle is excluded, leaving a three-dimensional image parameterization. Each position on the display is considered to be a multiview light source that can direct light in different (horizontal) directions. Each such 
multiview light source emits three wavelengths corresponding to Red Green and Blue (RGB) colors. The light emitted by the display is thereby given by:

$$
I=[R, G, B] ; R=R(x, y, v), G=G(x, y, v), B=B(x, y, v),
$$

where $x, y$ and $v$ are the position and direction coordinates. Each of these RGB-channels must be filtered in the same way in order to remove aliasing. We, therefore, simplify the notation to describe one wavelength only:

$$
I=I(x, y, v) .
$$

The samples can so be organized in ray-space according to horizontal, vertical and perspective positions. This spectrum can be visualized by considering one horizontal line at a time. Fig. 2 depicts this parameterization for one horizontal line $I=I(x, \cdot, v)$

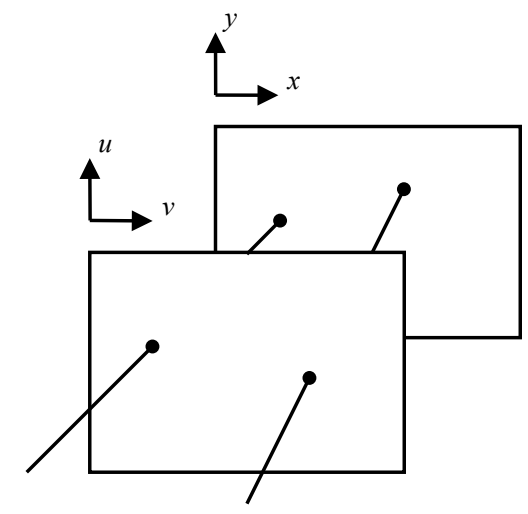

Figure 1. A light wave can be represented by the intersections of two planes. $(x, y)$ defines the position; $(u, v)$ defines the direction.
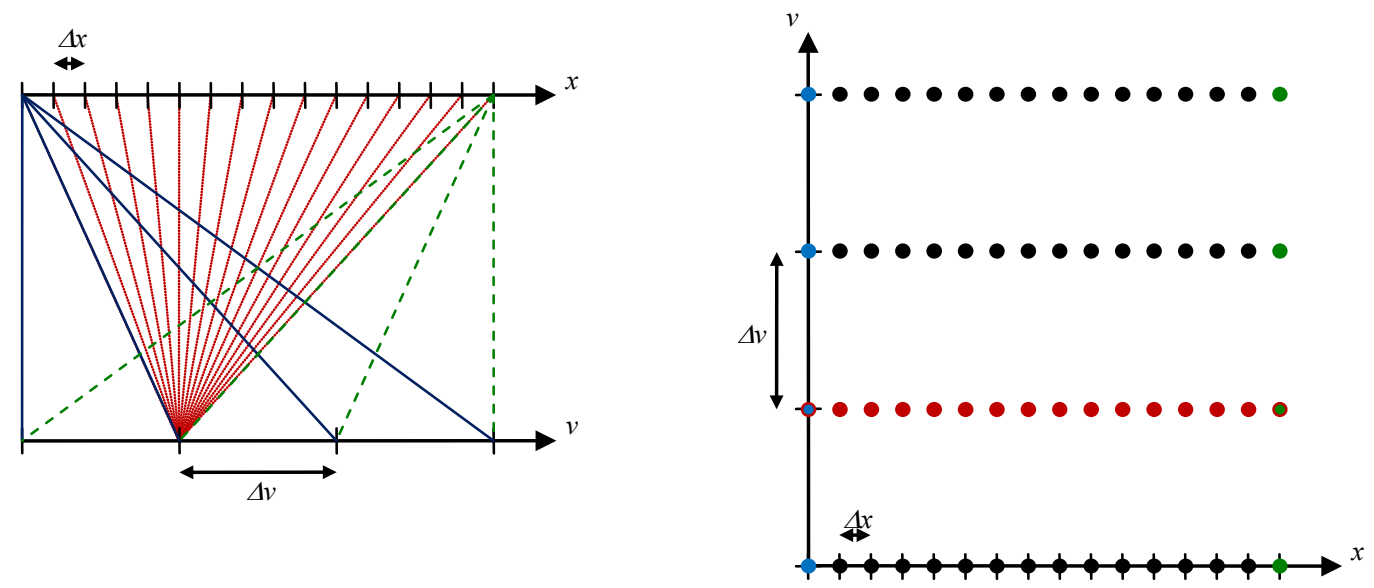

Figure 2. Horizontal cut of the two-plane representation and its corresponding ray-space.

\subsection{Ray-space dependence on depth}

A detail of the scene at a specific depth produces a line in the two-dimensional cut of the ray-space $I=I(x, ;, v)$. This is caused by the change in perspective as the view-parameter $v$ is altered. The gradient of this line is given by the disparity (in $x$-direction) between adjacent views. A detail at zero disparity (in the display plane) implies a vertical line as $\partial v / \partial x=\infty$. See Fig. 3. A detail at infinity implies an equal change in both $x$-direction and $v$-direction, leading to a gradient of $\partial v / \partial x=1$. Therefore, a detail positioned in the display zone ("behind" the display plane) has a gradient 
between 1 and infinity. A detail with negative disparity is in the viewer zone ("in front of" the display plane) and gives a negative gradient in ray-space.

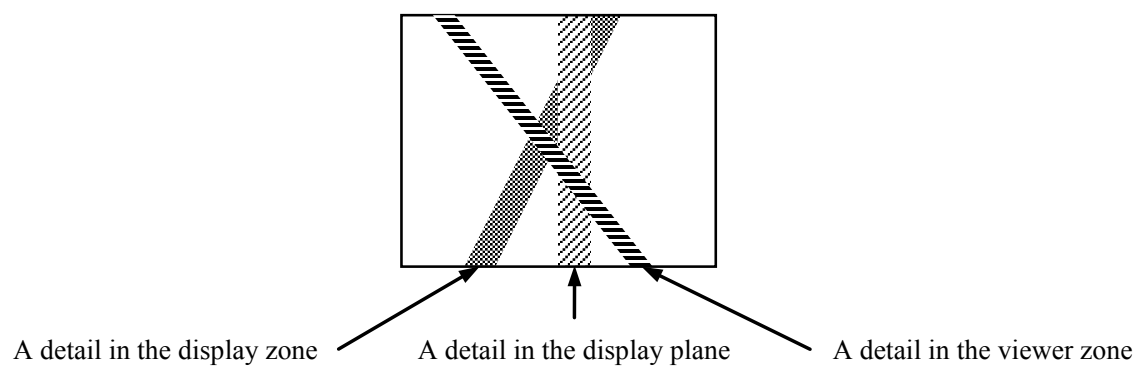

Figure 3. Gradients in ray-space depends on the disparity between adjacent views. Details in the display zone have a gradient between 1 and infinity. Details in the viewer zone have a negative gradient. Details in the display have a gradient equal to infinity. Note how closer details occlude farther details.

\subsection{Ray-space spectrum}

The construction of ray-space has consequences also in frequency domain. A straight line in ray-space becomes a straight lines passing through the origin in frequency domain. Details of zero disparity become a horizontal line, whereas details at infinity have a gradient equal to -1 in the ray-space spectrum. A negative disparity turns into a line with positive gradient in the frequency domain. See Fig. 4 . In the two-dimensional spectrum $\hat{I}\left(\omega_{x}, ; \omega_{v}\right)$, each detail so gives rise to lines passing through the origin, where the line orientation is defined by the distance to the zero-disparity plane (display plane). The frequency information of details in the scene is distributed between the limiting lines of objects farthest and closest in the scene.

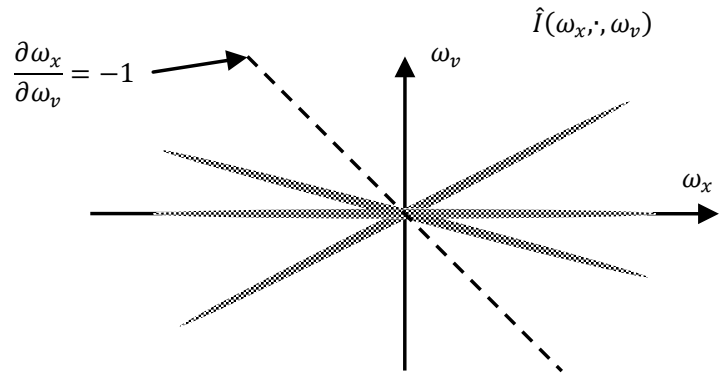

Figure 4. Ray-space spectrum of the continuous signal $\hat{I}\left(\omega_{x}, \omega_{v}\right)$. Details give rise to lines with an angle depending on the distance to display plane. A gradient of -1 means that details are at infinity.

\subsection{Sampling of ray-space}

A multiview display consists of a certain number of pixels that direct light into a certain number of views. In this way, the light emitted from the display is sampled both in position and direction.

In frequency domain, the information of the sampled multiview information $\hat{I}_{s}\left(\omega_{x}, \omega_{y}, \omega_{v}\right)$ equals the original spectrum superimposed with a period of the sampling frequency. In a multidimensional signal, the periodicity appears in each of the frequencies $\omega_{x}, \omega_{y}$ and $\omega_{v}$,

$$
\hat{I}_{s}\left(\omega_{x}, \omega_{y}, \omega_{v}\right)=\sum_{k=-\infty}^{\infty} \sum_{l=-\infty}^{\infty} \sum_{m=-\infty}^{\infty} \hat{I}\left(\omega_{x}-k \frac{2 \pi}{\Delta x}, \omega_{y}-l \frac{2 \pi}{\Delta y}, \omega_{v}-m \frac{2 \pi}{\Delta v}\right),
$$

where $\Delta x, \Delta y$ and $\Delta v$ are the sampling distances in each direction. As a consequence, an original (continuous) signal with high frequencies relative to the sampling frequency implies an overlap between the original spectrum and its replica that distorts the sampled signal. 
The inter-perspective aliasing (between views) is avoided when details are within a distance $d$ to the display plane such that $-D \Delta x /(\Delta v+\Delta x)<d<D \Delta x /(\Delta v-\Delta x)$. This corresponds to the slopes between the limiting slopes of $\partial \omega_{v} /$ $\partial \omega_{x}= \pm \Delta x / \Delta v$ in the ray-space spectrum ${ }^{13}$. Fig. 5 depicts the aliased areas when these limits are violated. The useful information is also indicated in the figure.

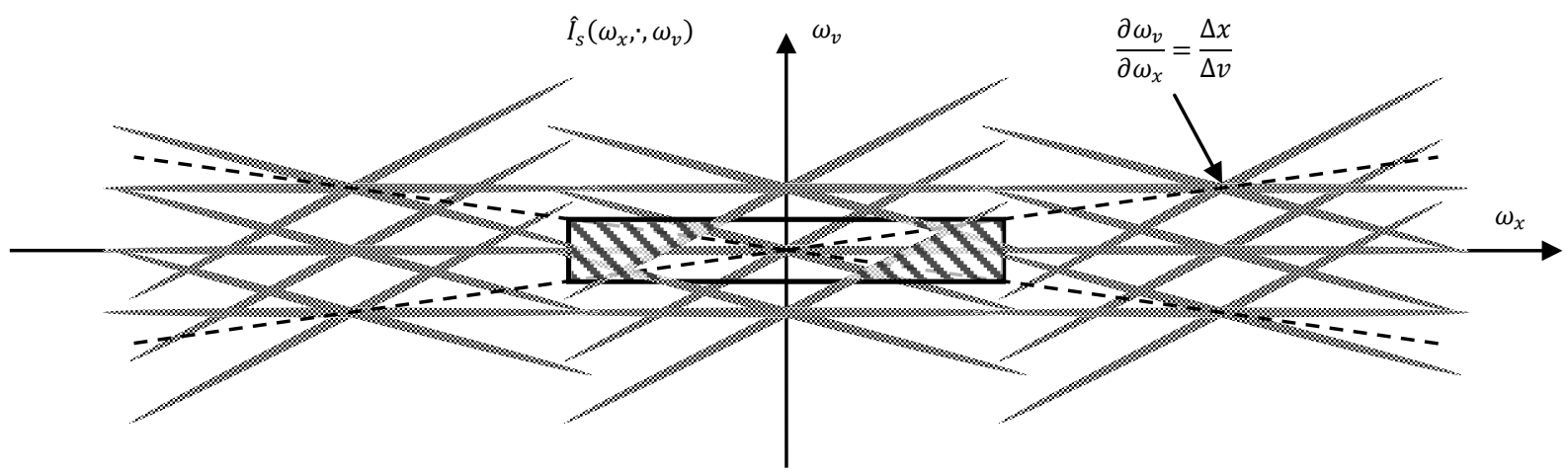

Figure 5. Ray-space spectrum of the sampled signal $\hat{I}_{s}\left(\omega_{x}, ; \omega_{v}\right)$. The center box indicates the Nyquist frequencies in both $x$ - and $v$-direction. Replicas imply inter-perspective aliasing when gradients exceed $\partial \omega_{x} / \partial \omega_{v}= \pm \Delta x / \Delta v$, marked with dashed lines. Frequencies with aliased information below the Nyquist frequencies are indicated by the shaded areas.

\section{PROPOSED METHOD}

A remedy to this aliasing in an already sampled signal is to filter it such that aliased frequencies are removed. We consider the three-dimensional ray-space signal line by line, $I=I(x, ; v) \forall y$. We propose to use a two-dimensional antialiasing filter that adapts the filter kernel to maintain alias-free information. Thus, the filter kernels are adjusted according to the frequency contents of the multiview data in order to avoid removing useful information unnecessarily. The filtering is performed in the ray-space (spatio-angular space), for which the FIR filter kernel is determined by the two-dimensional window method. Firstly, the filter transfer function is established from the proposed amplitude characteristics (see below), which are based on minimal and maximal depth in the multiview scene data. Secondly, a first filter kernel is obtained by the inverse Fourier transform. This first kernel is limited by multiplication with a rotationally symmetric Gaussian window. It reduces the size of the filter kernel and further smoothes edges in order to avoid frequency leakage at distinct borders.

\subsection{Locally content-dependent filter kernels}

The size of the filter amplitude characteristic is adjusted to the non-aliased parts. For each line the minimal and maximal depths are identified, and the filter amplitude characteristic is adapted to the frequencies limited by the lines defined by the depth range of the scene locally. See Fig. 6.
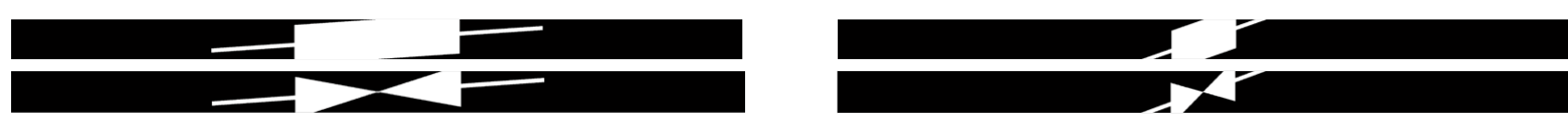

Figure 6. The size of the filter amplitude characteristics is adjusted to the depth range of the scene depth for each image line. The left images have less depth, the right more depth. The two top images depict the filter characteristics Ref 1 and the two bottom images depict Ref 2 .

\subsection{Depth detection}

The limiting slopes in the 2D ray-space spectrum are identified by linear searches in the amplitude spectrum. Firstly, the amplitude spectrum is transformed into logarithmic scale $\hat{I}_{\mathrm{s}, \log }\left(\omega_{x}, ; \omega_{v}\right)=\log _{10}\left(\hat{I}_{\mathrm{s}}\left(\omega_{x}, ; \omega_{v}\right)\right)$ and small values are set to zero according to a threshold. Secondly, the minimum and maximum angular frequency $\left(\omega_{v \text {,min }} ; \omega_{v \text {,max }}\right)$ for which the derivative $\partial \hat{I}_{\mathrm{s}, \log }\left(\omega_{x}, ; \omega_{v}\right) / \partial \omega_{v}>0$ are identified for two different given position frequencies on either side of the 
origin, $\omega_{x}= \pm \omega_{x 0}$. The slopes $k_{\min }$ and $k_{\max }$ so are computed from these four frequency points of the ray-space spectrum. See Fig. 7.

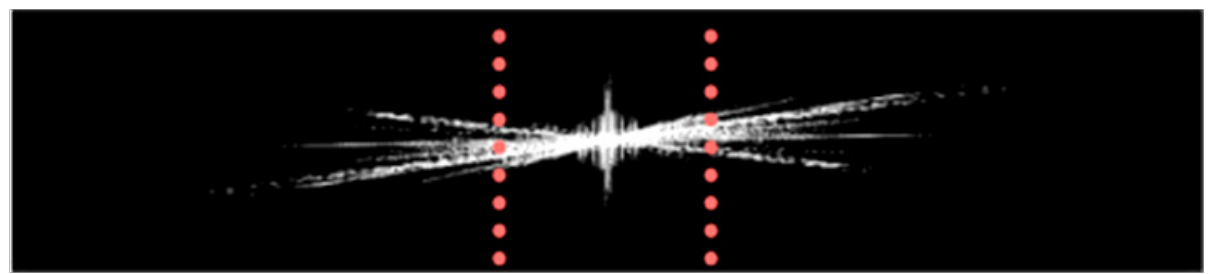

Figure 7. Depth detection method. The minimal and maximal slopes $k_{\min }$ and $k_{\max }$ of the spectrum are identified by linear search along the dotted lines.

\subsection{New filter kernels}

We proposed two new filter amplitude characteristics: Bowtie and Diamond. Both characteristics are limited by the slopes, $k_{\min }$ and $k_{\max }$, defined by the minimal and the maximal depths of the scene, and the aliased components in the spectrum due to these depths. See Fig. 8. The former follows the limiting slopes all along to the origin and takes the form of a bowtie. It so removes frequency components along the axis of zero angular frequency, which shall have no useful information. In order to assure inclusion of the zero frequency components, the shape is supplemented with a circle around the origin. The diamond shape includes more low frequency components by extending the characteristics from the crossing points of the limiting slopes.
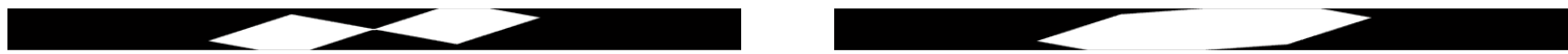

Figure 8. Proposed filter spectra characteristics. Bowtie (left) rejects all aliased information along with frequency components that shall contain no information. Diamond (right) rejects all aliased information but keeps more low frequency components. C.f. Fig. 6 (left) for the corresponding characteristics for Ref 1 and Ref 2.

\section{TEST ARRANGEMENT AND EVALUATION CRITERIA}

The proposed method was evaluated in two steps. Firstly, we evaluated the consequences of applying locally contentdependent filter kernels. The characteristics of the wide aperture reconstruction filter ${ }^{7}(\operatorname{Ref} 1)$ and of the combined preprocessing and reconstruction filter ${ }^{8}(\operatorname{Ref} 2)$ were used. See Fig. 6. Each of these content-adaptive filters was compared to its corresponding fix filter kernel. The limiting frequencies of the fixed filters were determined from the minimal and maximal depths of the whole multiview image. Secondly, we assessed the novel filter amplitude characteristics. The comparison was between Ref 1, Ref 2, Bowtie and Diamond. All of them applied locally contentdependent filter kernels.

\subsection{Parameter values and data set}

All filters and their testing were implemented in Matlab. The Gaussian window used $\sigma=15$ and a size of [90 x 90]. The multiview data set consisted of 40 perspective views of medical data of 1024 columns and 768 lines, rendered by raycasting from a voxel-based Computer Tomography scan. The number of views is in the range of a light field display ${ }^{1}$. The examined person is unknown; the data set was used to demonstrate an application area where a correct perception of the scene is required. The content of the data set is such that parts of it contain little depth, others large depth. Therefore, the assessment of different parts of the resulting views gives a good understanding for advantages and disadvantages of the investigated filters.

\subsection{Evaluation criteria}

We compared the resulting images in two different ways. Firstly, the amount of inter-perspective aliasing was evaluated with an ocular method. The filter outputs were compared by imitating a change between views: two adjacent views were superimposed, by which aliasing appears as duplets of details, i.e. details are repeated at different positions; no aliasing implies a smooth transition between the views so that details at large disparity are blurry and do not produce double images. Secondly, we compared the PSNR over all resulting perspective views using the different filter characteristics. 
The rationale for this choice is that PSNR gives a value of the similarity to the original data, and so judges the amount of data that have been removed, at the same time as the chosen filter amplitude characteristics assure no aliasing.

\section{RESULTS AND ANALYSIS}

The results of the filtering process are presented in Fig. 9 - 12, which show that all filters were good at reducing the aliasing effect.

\subsection{Fix versus content-dependent filter kernels}

Fig. 9 and 10 present the original data and the results of the filtering for both fix and content-dependent filter kernels; details of the images are enlarged. The filter amplitude characteristics of fix kernels are limited by the largest disparity of the whole data set. Therefore, fix kernels removed more information in the filtering process, also at positions $(x, y)$ far from the limiting details. As a consequence, fix kernels made the resulting images more blurry. The outputs of the content-dependent filters showed a clear improvement for details that had a shallow depth, i.e. were close to the zerodisparity plane. Especially the text at zero-disparity appeared much clearer, which is caused by higher cut-off frequencies (a larger shape filter spectrum) for that part of the image. The pointer-cross, with the most negative disparity, had about the same visibility for both the fix and content-dependent filters. This is due to the fact that both filter types apply the same filter kernel at that part of the image, i.e. the depth of the pointer-cross was the limiting factor for fix filter kernels. A slight improvement was also noticeable for details at an intermediate distance from the zero-disparity plane. Fig. 12 shows that all adaptive filters had higher PSNR, i.e. more data had been kept in the filtering process, yet no aliasing was present due to the filter design.

A closer look reveals, however, how lines containing large disparities were blurred for the full line, even if these large disparities only existed in a small portion of the line. This is a consequence of the line-by-line approach of the filter that selects a filter kernel based on the minimal and maximal depth for the full line.

\subsection{Comparison of filter amplitude characteristics}

In this section we compare the two reference filter amplitude characteristics, Ref 1 and Ref 2, and the two proposed ones, Bowtie and Diamond, when using the content-dependent filter method. See Fig. 9, 10 and 11.

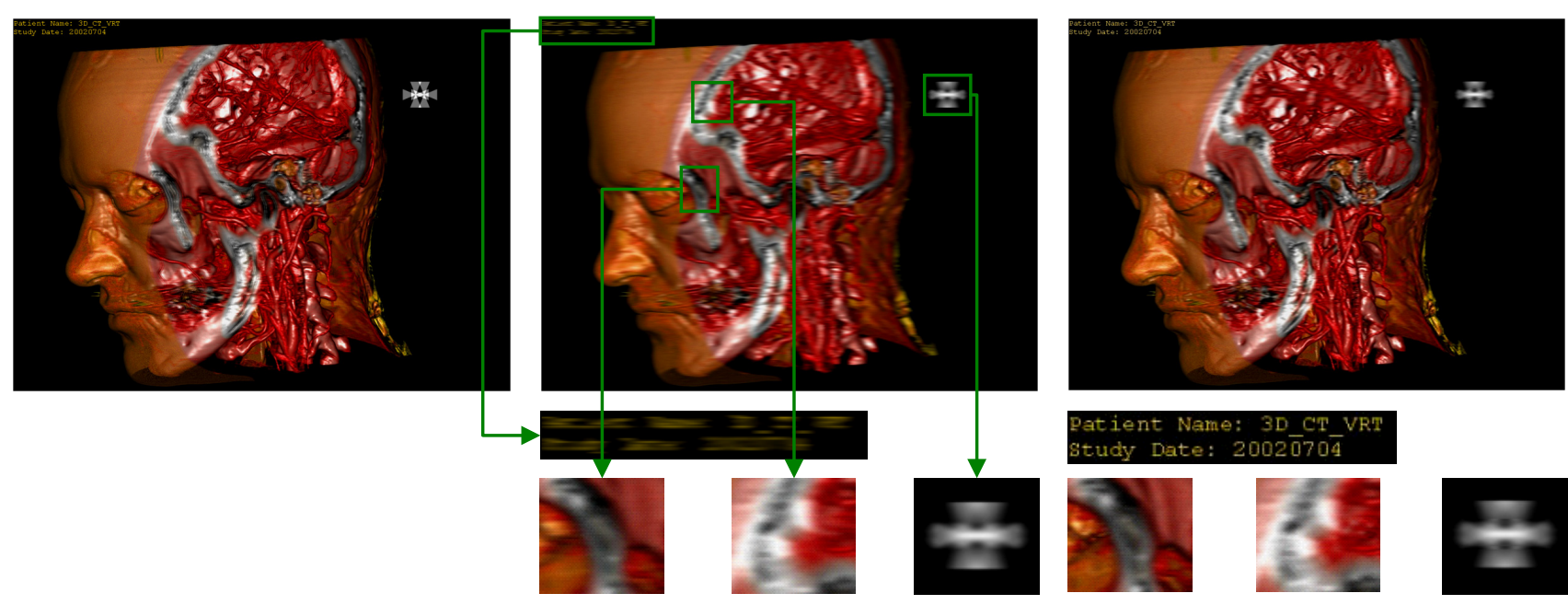

Figure 9. Superimposed adjacent views of original data (left) and of results after applying fixed (center) and contentdependent filter (right) kernels, using kernel Ref 1. Details of each image are presented below the corresponding result.

Both Ref 1 and Diamond had better results than Ref 2 and Bowtie, based on the PSNR diagram in Fig. 12, as well as the ocular inspection in Fig. 9 - 11. The outputs of the two former filters revealed more details, yet exhibiting no or minimal aliasing. Their difference to Ref 2 and Bowtie, respectively, is an inclusion of more frequencies in the regions along the angular frequency axis. Those regions do not correspond to any depth in the image, which was the actual reason for 
excluding those frequencies. Such frequencies constitute next to vertical lines in the ray-space (spatio-angular) spectrum, which imply $d v / d x=0$ in the multiview image, i.e. no change in the view (angular) direction but possible change in the horizontal direction. Such spectrum lines can therefore be caused by details stretching over large horizontal positions that can be viewed from many perspectives. The investigated multiview image did not contain such details. However, removing those frequencies by Ref 2 and Bowtie introduced horizontal stretches, see Fig. 10 and 11.
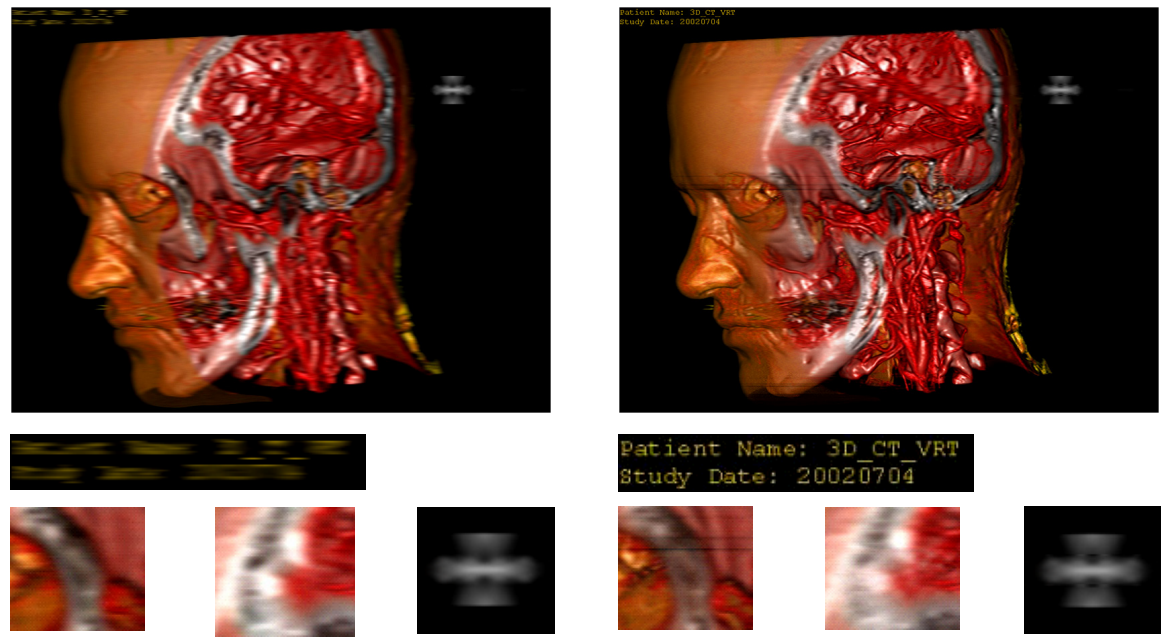

Figure 10. Superimposed adjacent views of results after applying fixed (left) and content-adaptable filter (right) kernels, using kernel Ref 2. Details of each image are presented below the corresponding result.
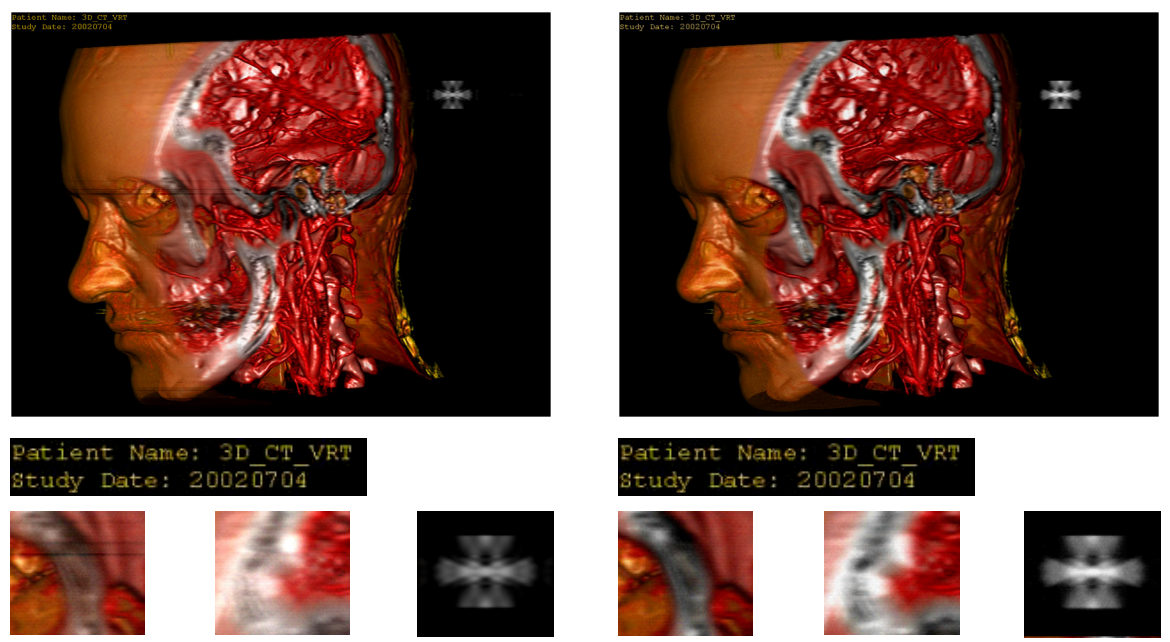

Figure 11. Superimposed adjacent views of results after applying content-adaptable filter kernels, using kernel Bowtie

(left) and Diamond (right). Details of each image are presented below the corresponding result.

The Ref 1 and Ref 2 filters imply removal of non-aliased information as their amplitude characteristics eliminate spatial frequencies $\omega_{x}$ above a certain limit along a vertical line, see Fig. 6 . This is the reason why the Ref 1 filter did not perform as well as Diamond. The best results were produced by the filter characteristic Diamond. According to the PSNR diagram in Fig. 12, it preserved the most information, and also produced the best results from an ocular inspection. This is a consequence of using the largest part of the signal filter spectrum of the investigated filters without including any frequencies with aliasing. 


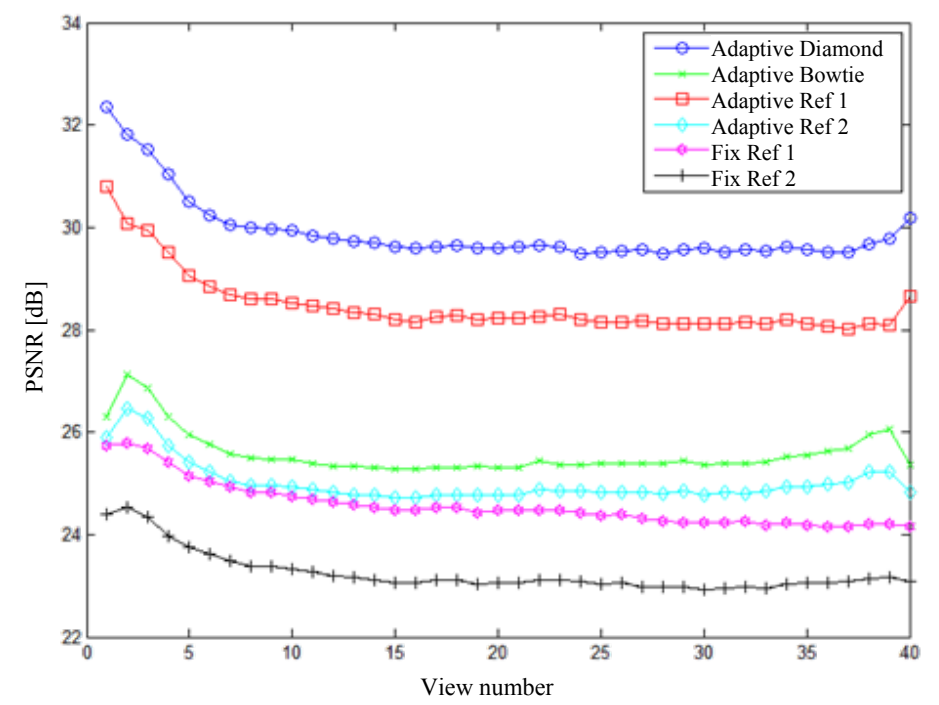

Figure 12. PSNR for filtered views relative the original views.

\section{CONCLUSIONS}

We have investigated inter-perspective aliasing caused by high frequency components in the spatio-angular ray space. We have proposed to adjust the filter kernels to local information of details' depths in the multiview data. We have further proposed new amplitude characteristics to include more non-aliased information in the filters, and a method to identify minimal and maximal depth from the ray-space spectrum. Different filter kernels have been compared.

The proposed method leads to alias-free multiview images of higher quality. Details at intermediate and small distances from the display plane are sharper applying the locally adjusted filter kernels, whereas details of larger disparity remains equally blurry as with fix filter kernels. The new filter characteristics Diamond included the most alias-free frequency components and so provided the highest quality and outperformed filters of earlier works.

These new inter-perspective aliasing-filters will lead to higher quality of multiview images on multiview and lightfield displays, and the method may become important in areas where a correct depth perspective is required such as science and medicine.

The study has demonstrated certain opportunities to further improvements of the filter design. Especially the line-by-line approach in the paper implied an uneven quality when details were at large differences in depth. Next we will study improved locality for the method, and refined filter parameters in order to improve quality.

\section{ACKNOWLEDGEMENTS}

This work has been supported by grant 2007/0390 of the Swedish Visualization programme (funded by the Knowledge Foundation, the Vårdal Foundation, Vinnova, Swedish Foundation for Strategic Research, and Invest in Sweden Agency), by grant 00156702 of the EU European Regional Development Fund, Mellersta Norrland, Sweden, and by grant 00155148 of Länsstyrelsen Västernorrland, Sweden. The authors would like to thank Assoc. Prof. Anders Persson at CMIV, Linköping University and Thomas Ericson, Setred AB, Sweden, for contributing and rendering the data used in this work. 


\section{REFERENCES}

[1] Setred AB, Surgery 3D display system, http://www.setred.com, dec (2011).

[2] Møller, Ch. M. and Travis, A. R. L., "Correcting Interperspective Aliasing in Autostereoscopic Displays," IEEE Trans. Visual. Comput. Graphics 11(2), 228-236 (2005).

[3] Jones, G. R., Lee, D., Holliman, N. S. and Ezra, D., "Controlling perceived depth in stereoscopic images", Proc. SPIE 4297, 42 (2001).

[4] Kim, M., Lee, S., Choi, C., Um, G.-M., Hur, N. and Kim, J., "Depth Scaling of Multiview Images for Automultiscopic 3D Monitors", Proc. 3DTV Conference Istanbul Turkey (2008).

[5] Chai, J.-X., Tong, X., Chan, X.-C. and Shum, H.-Y., "Plenoptic sampling," Proc. ACM SIGGRPH 2000, 307$318(2000)$.

[6] Isaksen, A., McMillan, L and Gortler, S.J., "Dynamically reparameterized light fields," Proc. ACM SIGGRPH 2000, 297-306 (2000).

[7] Stewart, J., Yu, J., Gortler, S.J. and McMillan, L., "A new reconstruction filter for under sampled light fields," Proc. 14th Euro graphics Workshop on Rendering, 150-156 (2003).

[8] Zwicker, M., Matusik, W., Durand, F. and Pfister, H., "Antialiasing for automultiscopic 3d displays," Rendering Techniques 2006: 17th Euro graphics Workshop on Rendering, 73-82 (2006).

[9] Zwicker, M, Yea, S., Vetro, A., Forlines, C., Matusik, W. and Pfister, H., "Display pre-filtering for multiview video compression," Proc. ACM Multimedia 2007, 1046-1053 (2007).

[10] Ramachandra, V., Hirakwa, K., Zwicker, M and Nguyen, T., "Spatio-angular prefiltering for multiview 3D displays," Trans. Visual. Comput. Graphics 17(5), 642-654 (2011).

[11] Gortler, A. J., Greszczuk, R, Szeliski, R, and Cohen, M. F., "The lumigraph," Proc. SIGGRAPH 96, 43-54 (1996).

[12]Levoy, M and Hanrahan, P. M., "Light field rendering," Proc. SIGGRAPH 96, 31-42 (1996).

[13]Zwicker, M., Vetro, A., Yea, S., Matusik, W. Pfister, H. and Durand, F., "Resampling, antialiasing, and compression in multiview 3-D displays," IEEE Sig. Proc. Mag. 24(6), 88-96 (2011). 